\title{
La Comunidad Andina en el contexto global Posreingeniería de 2014: una mirada desde la acción colectiva
}

\author{
Denny Piña García* \\ Giulliana Reggiardo Palacios**థ
}

\section{Resumen}

La Comunidad Andina (CAN) es un bloque de integración regional cuyos avances han sido siempre objeto de debate. En este sentido, el ensayo hace uso de la herramienta teórica de las ciencias políticas, específicamente, de la teoría de la acción colectiva, para el análisis del proceso de integración a partir de la reingeniería de 2014. Los resultados del análisis permiten determinar que cada integrante le otorga una valoración diferente a la identificación de los llamados bienes colectivos del bloque. Bajo esta lógica, esta valoración tiene un impacto directo sobre el éxito del organismo internacional en el marco de una globalización que, cada vez más, exige resultados conjuntos desde un enfoque de gobernanza.

Palabras claves: Comunidad Andina, acción colectiva, regionalismo, globalización, gobernanza, bienes colectivos, reingeniería.

\section{The Andean Community in the global context Post-reengineering 2014: a look from the collective action}

\section{Abstract}

The Andean Community (CAN) is a regional integration bloc and its progress has always been in debate. This essay uses the theoretical tools of the political sciences, specifically the theory of collective action, to analyze the integration process from the 2014 reengineering onwards. The results of the analysis allow to determine that each member gives a different valuation to the identification of the so-called collective assets of the bloc. Under this logic, this assessment has a direct impact on the success of the international organization in the context of a globalization that increasingly requires global results from a governance perspective

Keywords: Andean Community, collective action, regionalization, globalization, governance, public goods, reengineering.

* Funcionario en el Servicio Diplomático de la República del Perú. Maestría en Diplomacia y Relaciones Internacionales de la Academia Diplomática del Perú “Javier Pérez de Cuéllar. ‘dennypinagarcia@ gmail.com .

** Docente de la Universidad Peruana de Ciencias Aplicadas. Funcionaria en el Servicio Diplomático de la República del Perú. ‘g_reggiardo@hotmail.com〉.

Recibido: 16 de abril de 2020 | Revisado: 5 de agosto de 2020 | Aceptado: 19 de agosto de 2020 . Para citar este artículo: Piña García, Denny y Reggiardo Palacios Giulliana. "La Comunidad Andina en el contexto global. Posreingeniería de 2014: una mirada desde la acción colectiva”. Comentario Internacional 20 (2020): 79-100. doi: 10.32719/26312549.2020.20.1.5 


\section{Introducción}

a Comunidad Andina (CAN) es uno de los esquemas de integración regional más institucionalizados y sólidos en el mundo y, junto con la Unión Europea (UE), son los únicos bloques regionales de carácter supranacional ${ }^{1}$ que existen en la actualidad.

Si bien muchos autores señalan que nos encontramos en un periodo histórico marcado por la tendencia a un relacionamiento de carácter bilateral con los Estados, a nivel multilateral o regional, son destacables los avances realizados, concretamente, en el marco de la CAN, que ha cumplido medio siglo desde su creación y que está atravesando un proceso de reingeniería desde el 2014.

De acuerdo con las principales teorías de la corriente positivista de las relaciones internacionales, en términos generales, los Estados actúan racionalmente en función de sus intereses, entre los que se encuentran la seguridad, el desarrollo y el bienestar. Al respecto, estos elaboran e implementan políticas que permiten alcanzar los objetivos trazados.

En ese sentido, los esquemas de integración regional como la CAN tendrían que cumplir con alguna función específica de interés para sus Estados miembros, y su evolución solo es el resultado de la adecuación e implementación de políticas para cumplir con los objetivos que eventualmente satisfacen los intereses nacionales de los miembros.

Este trabajo realizará una aproximación heterodoxa al proceso de integración desde la perspectiva de las ciencias políticas considerando los aportes teóricos del concepto globalización, gobernanza e integración regional, para luego esbozar en su plena dimensión la teoría de la acción colectiva de Olson y que, consideramos, ofrece una perspectiva más amplia del proceso de integración andino. Debemos subrayar que el actual trabajo ha tomado como referencia una investigación precedente y muy completa de Casas y Correa $^{2}$ que entre sus enfoques metodológicos utiliza también la perspectiva de la teoría de Olson.

1. Para definición del concepto de "supranacionalidad" revisar Rafael Calduch, "Las organizaciones internacionales gubernamentales", en Relaciones Internacionales (Madrid: Ediciones Ciencias Sociales, 1991).

2. Andrés Casas y María Elvira Correa, "¿Qué pasa con la Comunidad Andina de Naciones - CAN?", Revista Papel Político 12, n. 2 (2007). 
El presente ensayo tiene por objetivo presentar brevemente un análisis de los avances de la reingeniería de 2014 de la CAN a la luz de la teoría de la acción colectiva. Todo ello con el fin de determinar la valoración que los miembros le otorgan a los bienes colectivos que crea el bloque y que son el resultado de sus intereses nacionales puestos en acción en el esquema de integración, bajo la metodología cualitativa-deductiva de la investigación científica.

\section{Globalización, gobernanza y regionalismo}

Globalización: ¿más caos a la anarquía?

Para una mejor comprensión de la evolución del proceso de integración de la CAN y de lo que representa, es necesario dilucidar en primer punto el fenómeno de la globalización como principal contexto donde participa el mencionado grupo. De hecho, la globalización es un término frecuentemente utilizado, no solo en el lenguaje político, sino también en los ámbitos económico y social. No obstante, aún existe debate académico respecto de los alcances del concepto mismo.

Para algunos autores contemporáneos, la globalización se refiere "a los procesos de cambio que sustentan una transformación en la organización de los asuntos humanos al unir y expandir la actividad humana en todas las regiones y continentes" y en "el grado en que la interconexión a través de las fronteras ya no es mera casualidad sino predecible y regularizada".

Asimismo, se puede considerar desde la perspectiva de Rosenau ${ }^{4}$ que, dentro de la conceptualización del término, no solo hace referencia a la internacionalización o aumento de interdependencia; sino, además, implica que la escala acumulativa, el alcance, la velocidad y la profundidad de la interconexión contemporánea están disolviendo la importancia de las fronteras y los límites que separan el mundo en sus numerosos Estados constituyentes o espacios económicos y políticos nacionales.

3. David Held et al., Global Transformations: Politics, Economics and Culture (Stanford: Stanford University Press, 1999), 15.

4. James Rosenau, Along the Domestic-Foreign Frontier: Exploring Governance in a Turbulent World (Cambridge, UK: Cambridge University Press, 1997). 
A pesar de que diversos académicos no están de acuerdo sobre las fuerzas causales que genera la globalización, la mayoría concuerda en que debe concebirse como un proceso relativamente largo y debe entenderse como un proceso múltiple, ya que la desterritorialización, la interconexión social y la aceleración se manifiestan en muchos ámbitos diferentes económicos, políticos y culturales de la actividad social. Cabe precisar que, de acuerdo con Scheuerman, ${ }^{5}$ cada manifestación de la globalización también genera distintos conflictos y dislocaciones.

Adicionalmente, desde la academia existen diferentes perspectivas sobre sus implicancias. Para los realistas, por ejemplo, la globalización no altera la característica más importante de la política mundial, es decir, la división territorial del mundo en Estados nación. La globalización puede afectar nuestras vidas a nivel social, económico y cultural, pero no trasciende el sistema político internacional de los Estados, el cual se sustenta en los Estados nación y en sus capacidades.

Por otro lado, para los liberales, la globalización es un producto final de una transformación a largo plazo de la política mundial. Para ellos, este fenómeno socava fundamentalmente los relatos realistas de la política mundial, al mostrar que los Estados no son los actores centrales como lo fueron antes y, en su lugar, existen múltiples actores de diferente importancia. Los liberales están particularmente interesados en la revolución en tecnología y comunicaciones representada por la globalización. Este aumento de la interconexión entre sociedades, que está dirigida por la economía y la tecnología, da como resultado un patrón muy diferente de las relaciones políticas mundiales con respecto al escenario anterior.

Para los marxistas, la globalización es un poco falsa. No es un proceso nuevo, y es considerado como la última etapa en el desarrollo del capitalismo internacional. Para los teóricos constructivistas, la globalización tiende a presentarse como una fuerza externa que actúa sobre los Estados, que los líderes a menudo sostienen que es una realidad que no pueden desafiar. En ese orden de ideas, los constructivistas piensan que podemos moldear la globalización en una variedad de formas, especialmente porque nos ofrece

5. William Scheuerman, "Globalization", The Stanford Encyclopedia of Philosophy winter 2018 Edition, (2018). 
oportunidades muy reales de crear movimientos sociales transnacionales ayudados por formas tecnológicas modernas de comunicación. ${ }^{6}$

Con respecto a las escuelas más modernas de las relaciones internacionales como los postestructuralistas, la globalización es considerada tan solo un discurso. Ellos son escépticos sobre las grandes afirmaciones hechas por realistas, liberales y marxistas sobre la naturaleza de la globalización, pues argumentan que cualquier afirmación sobre el significado de esta solo tiene sentido en el contexto de un discurso específico, producto del poder. No obstante, la erudición poscolonial sobre la globalización es similar a gran parte del pensamiento marxista en el sentido que destaca el importante grado de continuidad y persistencia de las formas coloniales de poder en el mundo globalizado. ${ }^{?}$

Sin embargo, la globalización plantea un desafío fundamental para cada uno de estos esquemas teóricos tradicionales. No es evidente que los Estados nación pueden describirse como esquemas de cooperación autosuficientes para todos los propósitos esenciales de la vida humana en el contexto de una intensa desterritorialización y la difusión e intensificación de las relaciones sociales a través de las fronteras, como si estas dejarán de tener significado concreto.

\section{Gobernanza: un orden dentro del caos}

Los problemas comunes que la globalización ha traído o acelerado, generan retos para la metodología de respuesta, porque son contados los casos en que los Estados pueden resolverlos por sí mismos.

La gobernanza en palabras de autores como Thomas Weiss ${ }^{8}$ es el resultado de la vinculación de los procesos de globalización e interdependencia. En ese sentido, la percepción de una tendiente y cada vez mayor desterritorialización hace necesario alguna forma de atención a los problemas que no pueden ser resueltos por las capacidades individuales de un determinado actor, sustancialmente los Estados.

6. John Baylis, Steve Smith, y Patricia Owens, The Globalization of World Politics: An Introduction to International Relations (Oxford: Oxford University Press, 2014).

7. Baylis, Smith, y Owens, The Globalization of World Politics.

8. Thomas Weiss, Global Governance: Why? What? Whither? (New York: Polity, 2013). 
Por lo tanto, la gobernanza es el concepto por el cual se determina "el conjunto de valores, reglas, normas, prácticas y organizaciones formales e informales que proveen un mejor orden"; 9 es decir, el marco conceptual que permite brindar orden al caos derivado de la globalización.

Pero lo importante es lo que establece este marco conceptual: el reconocimiento de la capacidad o legitimidad para resolver problemas globales o regionales. Siendo esto así, el típico ejemplo de gobernanza son las organizaciones internacionales, que no son otra cosa que aquellas estructuras formales que buscan proveer de bienes públicos. ${ }^{10}$ Es claro, como señala Barkin, ${ }^{11}$ que la mayoría de las organizaciones existentes no son de escala global o mundial, sino más bien esquemas regionales que buscan resolver conflictos o problemas de un determinado espacio geográfico.

Tradicionalmente, las organizaciones internacionales son definidas como intergubernamentales, precisamente porque su naturaleza está determinada por sus miembros que son los Estados, sin restarle capacidades a los mismos para conseguir sus objetivos en el escenario internacional. Pero existen algunas organizaciones que superan este concepto, y como se ha referido previamente terminan siendo supranacionales. Además de la UE, la CAN es un esquema de integración creado bajo el concepto de supranacionalidad que busca generar una gobernanza en su región para el beneficio de sus Estados miembros, y de esa forma resolver problemas derivados de la globalización que de otra forma no podrían ser resueltos. Es decir, buscan proveer bienes colectivos para el logro de sus objetivos.

\section{Regionalismo: ¿una respuesta conjunta para el contexto actual?}

Expuesto todo lo anterior, "la integración regional ${ }^{12}$ es una respuesta a la globalización, en su contrapartida, el recurso de conjugar las potenciali-

9. Ibíd., 31.

10. Samuel Barkin, International Organization: Theories and Institutions (New York: Pallgrave Mcmillian, 2013).

11. Ibíd.

12. La integración regional formal se refiere a procesos mediante los cuales los Estados van más allá de la eliminación de obstáculos a la interacción entre sus países y crean un espacio regional sujeto a algunas reglas comunes distintas. Con respecto a la integración económica, generalmente se distinguen varios grados de integración, de acuerdo con Belá Balassa: área de libre comercio, unión aduanera, mercado común, unión económica y monetaria. Baylis, Smith, y Owens, The Globalization of World Politics. 
dades nacionales para hacer frente al desafío internacional" ${ }^{13}$ Es decir, para estos autores, el regionalismo puede considerarse como uno de los pocos instrumentos disponibles para que los Estados puedan controlar los efectos - positivos o negativos- de la globalización. Si los Estados individualmente ya no tienen capacidad efectiva de regulación frente a los movimientos incontrolados de capital, cambio climático y otros problemas globales, entonces el regionalismo puede recuperar cierto control sobre estas consecuencias negativas.

En ese sentido, el regionalismo es un aspecto del proceso de globalización que puede concebirse como una forma de gobernanza localizada en una determinada región geográfica. Los avances de estos esquemas retroalimentan los desarrollos en otras. Por otra parte, dentro de la tendencia global del regionalismo, existen diferencias importantes en los tipos de organización establecidos, que van desde acuerdos flexibles y no vinculantes, como sucede con la Alianza del Pacifico (AP), la Asociación de Naciones del Sudeste Asiático (ASEAN) o inclusive el Foro de Cooperación Económica Asia-Pacífico (APEC); hasta la compleja arquitectura institucional, donde se puede destacar en cierta medida, el Mercado Común del Sur (Mercosur) o la Asociación Europea de Libre Comercio (EFTA), la Unión Africana o la UE y la CAN, dependiendo del alcance y la profundidad con el cual los miembros buscan abordar temas de gobernanza transnacional.

A inicios del presente año, una pandemia que se desató en China colapsó los sistemas sanitarios de varios países del mundo. Muchos analistas, entre ellos Diana Tussie, ${ }^{14}$ señalan que, probablemente, habrá una resistencia a la globalización debido a que antes de la crisis del Covid-19 existía una ola de nacionalismos en diferentes lugares del mundo y que, esto puede incrementarse a raíz del efecto dominó que genera el coronavirus y que se entiende como el resultado del propio proceso de globalización. Sin embargo, donde unos ven crisis, otros ven oportunidades y, tal vez, este es el momento para el regionalismo, como mecanismo de atención de problemas existentes y una forma de una globalización más benigna.

Asimismo, dentro de las teorías más recientes de integración regional encontramos teorías como el neofuncionalismo, la cual es una aproxima-

13. Baylis, Smith, y Owens, The Globalization of World Politics, 404.

14. Diana Tussie, "El coronavirus va a profundizar la resistencia a la globalización", La Nación, 5 de abril de 2020. 
ción teórica que ha evolucionado a lo largo de las últimas décadas. Al respecto, uno de sus más recientes exponentes, Francesco Nicoli ${ }^{15}$ quien, en su última revisión de la teoría, la concibe como una perspectiva integral de la integración dirigida por las crisis. El mismo autor identifica como limitantes a esta teoría, la falta de análisis sobre las preferencias de los Estados miembros individuales y de los grupos sociales -en el marco de la UE-, tanto en el plano interno, como externo e interregional y, como finalmente estas preferencias influyen en los resultados $;{ }^{16}$ siendo esto así, las decisiones que se tomaron durante la crisis de la integración europea no tomaron en consideración estas variables.

Es precisamente en este punto, donde podemos afirmar que los conceptos tradicionales de integración terminan siendo insuficientes, porque no salen del marco descriptivo de la metodología científica, ${ }^{17}$ haciendo necesario que, para un análisis más profundo y que esté acorde con las necesidades de la época, se haga uso de otras teorías, como las de la ciencia política, tradicionalmente no utilizadas pero que permitirán encontrar una razón detrás del funcionamiento o no del proceso de integración de la CAN.

\section{La Comunidad Andina y la reingeniería de 2014: ¿un proceso en evolución?}

La historia de la CAN comenzó a gestarse el 16 de agosto de 1966, cuando los presidentes de Colombia, Chile y Venezuela, y los delegados de los presidentes de Ecuador y Perú, reunidos en la capital colombiana, firmaron la Declaración de Bogotá, en la que se sentaron las bases del proceso de integración. ${ }^{18}$

El 25 de mayo de 1969 se aprobó el Acuerdo de Cartagena o Pacto Andino, documento constitutivo de la organización de integración regional. Es claro que, como señalan Casas y Correa, ${ }^{19}$ durante la primera década de su funcionamiento, la CAN, se centró en su desarrollo, pero algo que

15. Franceso Nicoli, "Neofunctionalism revisited: Integration Theory and varieties of outcomes in the Eurocrisis", Journal of European Integration 41, (2019).

16. Ibíd.

17. Casas y Correa, “Qué pasa con la Comunidad Andina de Naciones-CAN?".

18. Ibíd.

19. Ibíd. 
no abordan directamente es que este proceso era inspirado por las teorías regionalistas de la época. Estas teorías respondían a los inicios de lo que después fue el proceso de globalización. Con vaivenes y avances, el proceso de integración no generó mayores desarrollos durante la siguiente década y quedó estancado.

En 1990, los presidentes de los países andinos relanzaron el proceso de integración. En 1994 se anunció un Arancel Externo Común (AEC) y pasó a denominarse Comunidad Andina (CAN) en 1997, con el objetivo de consolidar un mercado común para 2005. El sistema institucional se basó en la Comunidad Europea, con elementos formales del supranacionalismo: las normas andinas deben ser directamente aplicables y disfrutar de la primacía sobre la ley nacional, y son monitoreadas por instituciones comunes, incluido un Tribunal de Justicia. ${ }^{20}$

Las nuevas formas de integración se consideraron fundamentalmente diferentes, como parte de reformas estructurales de amplia base, destinadas a encerrar compromisos en un contexto de liberalización unilateral y multilateral. También parecía que podría haber una nueva convergencia de iniciativas hemisféricas y latinoamericanas, ${ }^{21}$ que en cierta forma eran similares al proceso que originó la CAN en la década de 1970.

En el año 2012, los países miembros de la CAN participaban poco en cadenas de valor globales y los avances dentro de este esquema de integración eran cuestionados. Esta situación se dio incluso en el comercio intrarregional, pese a su mayor contenido de manufacturas. Además, acontecimientos como los Tratados de Libre Comercio firmados por Colombia y Perú, la salida de Venezuela y, la intención, ahora concretada, de Bolivia de pertenecer al Mercosur, confirmaron que la pretensión de la Unión Aduanera en la CAN pasará de ser una situación imperfecta a prácticamente una ficción. De hecho, la Decisión 669, de agosto de 2007, suspendió el AEC originado en la anterior Decisión 370.

El proceso de reingeniería del año 2014 tuvo lugar en un entorno de importantes cambios en el panorama de la integración económica latinoamericana y sudamericana. Reflejo de ello es el hecho de que los cuatro países miembros de la CAN (Bolivia, Colombia, Ecuador y Perú) perte-

20. Baylis, Smith, y Owens, The Globalization of World Politics, 407.

21. Ibíd. 
necen también a otros esquemas de integración económica, con diversas orientaciones de política.

Conforme la Decisión 792 del 23 de septiembre de 2013, los temas prioritarios incluyen: Restructurar el Sistema Andino de Integración para adecuarlo a las nuevas realidades y retos del contexto internacional; aspectos sociales de la integración: a) Promover que los ciudadanos puedan viajar, trabajar, estudiar y residir en cualquier país andino sin discriminación por la nacionalidad, considerando las particularidades y la seguridad interna de los Países Miembros. b) Promover la identidad andina. 3) Profundizar la integración comercial: a) Internacionalización de las MIPYMES. b) Mecanismos para incrementar el comercio intracomunitario, tales como: i) Elevación y armonización de estándares de calidad y reconocimiento mutuo de registros sanitarios. ii) Facilitación del comercio: aduanas, obstáculos técnicos al comercio. c) Exportaciones con valor agregado y encadenamientos productivos. d) Transporte internacional de pasajeros y mercancías. e) Promoción comercial conjunta. 4) Interconexión eléctrica: a) interconexión intra-regional. b) Interconexión con otros países de la región. 5) Respecto a una eventual y gradual articulación, complementación y convergencia de acciones en el marco de la CAN, Mercosur y Unasur.

Dentro de los beneficios que presenta la Comunidad Andina resalta su carácter supranacional y vinculante, es un espacio para el libre comercio de bienes que ha logrado avances en ciudadanía andina y libre movilidad de trabajadores migrantes y ha sido pionero en ámbitos distintos del económico-comercial (temas ambientales, pueblos indígenas, participación de la sociedad civil, entre otros).

En ese orden de ideas, los principales resultados de la Comunidad Andina son el orden jurídico andino que permite a los gobiernos adoptar normas comunitarias de cumplimiento obligatorio y políticas en temas de interés común; así como, la libre circulación de los productos de origen andino sin pagar aranceles. Esto contribuye a que el intercambio comercial en la subregión esté compuesto mayoritariamente por productos manufacturados que son los que generan más empleo. Cabe precisar que, en el documento Cifras CAN 2019, el 78\% de las exportaciones intercomunitarias corresponden a productos manufacturados; así como, el 93\% de las importaciones de la CAN desde el mundo. El mercado andino fue el segundo destino de las exportaciones de productos manufacturados de los países miembros de la CAN, con una participación del 13,7\% del total. 
Adicionalmente, cabe destacar los derechos ciudadanos como viajar con documento de identidad, sin necesidad de visa ni pasaporte; ${ }^{22}$ la implementación electrónica de la Tarjeta Andina de Migración ${ }^{23}$ y los avances en el marco regulatorio para la interconexión subregional de sistemas eléctricos e intercambio intracomunitario de electricidad. ${ }^{24}$

Recientemente, se pueden citar los logros hasta la fecha de los avances del bloque tales como, la puesta en órbita del satélite SES-10, una estrategia conjunta para la gestión de riesgos y desastres $;^{25}$ el régimen de propiedad intelectual que protege las creaciones del intelecto; ${ }^{26}$ sistemas andinos de calidad, ${ }^{27}$ sanidad agropecuaria ${ }^{28}$ y sanidad humana para proteger la salud de los consumidores. ${ }^{29}$

En vista de lo señalado en el cuarto punto de la Declaración ministerial de Cartagena de Indias con motivo de la celebración de los 50 años de la Integración Andina, existe la "voluntad de continuar trabajando de manera coordinada entre los diferentes Órganos e Instituciones del Sistema Andino

22. Comisión de la Comunidad Andina, Decisión 503 sobre el reconocimiento de documentos nacionales de identificación, 31 de diciembre de 2005.

23. Decisión 397 sobre la tarjeta Andina de Migración (TAM), 16 de septiembre de 1996.

24. Decisión 536 sobre el marco general para la interconexión subregional de sistemas eléctricos e intercambio intracomunitario de electricidad, 19 de diciembre de 2002; Decisión 757 sobre la vigencia de la Decisión 536, 22 de agosto de 2011; Decisión 816 sobre el marco regulatorio para la interconexión subregional de sistemas eléctricos e intercambio intracomunitario de electricidad, 24 de abril de 2011.

25. Decisión 672 sobre la modificación de la Decisión 654 sobre el "Marco Regulatorio para la Utilización Comercial del Recurso Órbita Espectro de los Países Miembros", 21 y 28 de septiembre de 2007; Decisión 725 sobre la autorización comunitaria para la explotación y comercialización del Recurso Órbita Espectro de los Países Miembros en la posición $67^{\circ}$ Oeste. 10 de diciembre de 2009

26. Decisión 351 el régimen común sobre el derecho de autor y derechos conexos, 17 de diciembre de 1993; Decisión 486 sobre el régimen común sobre propiedad industrial. 14 de septiembre de 2000; Decisión 345 sobre el régimen común de protección a los derechos de los obtenedores de variedades vegetales, 21 de octubre de 1993; Decisión 391 sobre el régimen común sobre acceso a los recursos genéticos, 2 de julio de 1996; Decisión 291 sobre el régimen común de tratamiento a los capitales extranjeros y sobre marcas, patentes, licencias y regalías, 21 de marzo de 1991; Decisión 632 sobre la aclaración del segundo párrafo del artículo 266 de la Decisión 486 de 2000, 6 de abril de 2006; Decisión 689 sobre la adecuación de determinados artículos de la decisión 486, 13 de agosto de 2008; Decisión 366 sobre la modificación de la disposición transitoria tercera de la Decisión 345. 26 de noviembre de 1994; Decisión 423 sobre la modificación de la octava disposición transitoria de la decisión 391, 11 de noviembre de 1997; Decisión 448 sobre la modificación de la octava disposición transitoria de la decisión 391, 11 de diciembre de 1998.

27. Decisión 376 sobre la aplicación de las Tablas Aduaneras del Sistema Andino de Franjas de Precios, 18 de abril de 1995; Decisión 419 sobre la modificación de la Decisión 376, 30 de julio de 1997.

28. Decisión 515 sobre el sistema andino de sanidad agropecuaria, 8 de marzo de 2002.

29. Decisión 516 sobre la armonización de legislaciones en materia de productos cosméticos, 8 de marzo de 2002; Decisión 706 sobre la armonización de legislaciones en materia de productos de higiene doméstica y productos absorbentes de higiene personal, 9 y 10 de diciembre de 2008. 
de Integración para el logro de los objetivos de la CAN”. Con referencia al proceso de reingeniería de la CAN, en el décimo primer punto se manifiesta "la importancia de concluir el proceso de reingeniería del Sistema Andino de Integración, particularmente las recomendaciones del Grupo de Trabajo sobre la Universidad Andina Simón Bolívar".

Del mismo modo, el noveno punto de la Declaración del Consejo Presidencial Andino realizada el 8 de junio de 2020, refiere a la necesidad de que los Países Miembros:

realicen los esfuerzos necesarios para finalizar los compromisos derivados del Proceso de Reingeniería de la CAN, particularmente sobre la reforma del Sistema Andino de Solución de Controversias y el Tribunal Andino de Justicia, así como lograr los consensos que permitan la consolidación institucional de la Universidad Andina Simón Bolívar y su fortalecimiento.

Siendo esto así, podemos identificar que el proceso de reingeniería sigue en una etapa continua de diseño e implementación que aún no ha concluido.

Adicionalmente, en el actual contexto de la emergencia sanitaria del Covid-19, la Declaración del Consejo Consultivo Empresarial Andino del 4 de mayo de 2020, hace énfasis en que "los retos y desafíos de la coyuntura actual que enfrentan los Países Miembros de la CAN a causa del COVID-19, y en el entendido que el proceso poscoronavirus requiere de una Comunidad Andina fortalecida". Es decir, este órgano del Sistema Andino de Integración reconoce la importancia del fortalecimiento del bloque, objetivo principal del inicio de la reingeniería del 2014, para atender situaciones como la actual emergencia sanitaria.

De igual forma, en el Protocolo sobre medidas de prevención sanitaria para las operaciones de Tránsito Aduanero Comunitario entre los Países Miembros de la Comunidad Andina como consecuencia de la pandemia del COVID-19 el 2 de mayo de 2020, se puede verificar la importancia de la institucionalidad de la CAN como un espacio para responder a la emergencia sanitaria en los países miembros y no afectar las cadenas de suministro, a través del uso de copias electrónicas de documentos aduaneros, lo que significa un avance importante en el proceso de integración.

Por otro lado, a pesar de los relevantes logros obtenidos luego del inicio del diseño e implementación de la reingeniería que se citan en los párrafos precedentes, es importante subrayar que persisten los principales problemas 
que ha presentado la CAN a lo largo de su historia. Siendo esto así, las teorías usuales han argumentado que se debe al bajo nivel de interdependencia entre sus miembros, la agenda y disponibilidad de recursos, e inclusive la falta de voluntad política, pero como se ha hecho hincapié en este ensayo, la principal hipótesis es que un análisis a la luz de la teoría de acción colectiva permitirá dilucidar que el problema es mucho más primigenio.

\section{La teoría de la acción colectiva: ¿una aproximación racional para entender los desafíos de la CAN?}

\section{Una mirada a la CAN desde la Teoría de la Acción Colectiva}

Teniendo en cuenta las anteriores aproximaciones teóricas, este estudio ha permitido hacer uso de la teoría de la acción colectiva como perspectiva que permitirá comprender la CAN de una forma heterodoxa, pero necesaria en el contexto posreingeniería del 2014. La razón es que se considera que el punto de partida más racional, en un nivel teórico y de acuerdo con las ciencias políticas para analizar las organizaciones, es su finalidad. Este principio aplica para todo tipo de organización, inclusive las de naturaleza económica. ${ }^{30}$ Aunque es difícil que una organización tenga una finalidad única, es una tendencia principal que la finalidad sea el proteger los intereses de sus miembros. ${ }^{31}$ Esta lógica, se ajusta también al bloque regional de la CAN en la medida que no solo es una organización de naturaleza económica sino también que desde su creación -con el Acuerdo de Cartagena- ha determinado objetivos que se pueden resumir en conseguir el desarrollo de sus miembros. Este objetivo perdura en el listado de temas priorizados en la reingeniería de 2014, lo que corrobora que la CAN es una organización con una finalidad específica.

La legitimidad de una organización se otorga en la medida que protege los intereses de sus miembros, esto implicaría que las organizaciones puedan desempeñar una función cuando existan estos intereses que se identifican

30. Mancur Olson, La lógica de la acción colectiva: Bienes Públicos y la Teoría de Grupos (México: Limusa Noriega Editores, 1992), 15.

31. Ibíd. 
como comunes o de grupo. ${ }^{32}$ En tanto, para la teoría de la acción colectiva, no tendría objeto tener una organización cuando la acción individual no organizada puede servir a los intereses del actor igual o mejor que la organización. ${ }^{33}$ La CAN en sus objetivos establece la necesidad de conseguir el desarrollo para sus Estados miembros, esta finalidad por sí misma le otorga legitimidad de existencia porque es una conducta racional de maximización de beneficios. ${ }^{34}$

En ese sentido, ¿cuáles son los fundamentos que permitirían que un conjunto de actores pueda proveerse de bienes colectivos sin recurrir a la coacción ni a otros estímulos positivos aparte del bien colectivo mismo, es decir, tengan un interés o intereses comunes? ${ }^{35}$ Según la teoría referenciada existen tres factores acumulativos pero diferenciados que permiten analizar por qué ciertos grupos no pueden accionar colectivamente de forma eficiente $^{36} y$, por qué el tipo de organización que cumple con estos criterios, tienen mayor factibilidad de tener intereses comunes.

\section{La racionalidad para entender} los desafíos de la CAN

El primer factor refiere al tamaño de la organización, mientras más grande la organización, más pequeña la fracción del beneficio real que el individuo recibirá de actuar en beneficio del interés común. ${ }^{37}$ La CAN como destacan Casas y Correa $^{38}$ se puede catalogar como un grupo pequeño porque está conformado por solo cuatro miembros -Bolivia, Colombia, Ecuador y Perú- y cumpliría con este presupuesto teórico de la acción colectiva.

El segundo factor considera que, dada esta condición -los grupos pequeños-, es poco probable que alguno de los actores tenga incentivos para asumir los costos de proveer una pequeña porción del interés común cuando el grupo es grande. El tercer factor establece que, mientras más actores participen de una organización, mayores serán los costos de sostenimiento

32. Ibíd., 17.

33. Ibíd.

34. Casas y Correa, "¿Qué pasa con la Comunidad Andina de Naciones-CAN?".

35. Olson, La lógica de la acción colectiva: Bienes Públicos y la Teoría de Grupos, 43.

36. Ibíd., 48.

37. Ibíd.

38. Casas y Correa, "iQué pasa con la Comunidad Andina de Naciones-CAN?". 
de la organización. ${ }^{39}$ El problema fundamental con la CAN es que a pesar de que cumple el primer presupuesto, parecería como señalan Casas y Correa ${ }^{40}$ que la lógica de la acción colectiva opera a la inversa en el bloque regional; en tanto, un análisis no solo de su historia y las razones que llevaron a la reingeniería del 2014, hacen ver que la organización a pesar de tener un número reducido de miembros funciona como si se tratara de una organización grande. ¿A qué se debe esto?

Las condiciones que permiten el sostenimiento de la acción colectiva de acuerdo con la teoría de Olson, son: el tamaño reducido de los miembros, el interés o intereses comunes de los actores y, los incentivos para cooperar en búsqueda del interés o intereses comunes. La teoría desarrollada por su autor en 1965 incluyó la determinación de grupos vinculados a la recepción y disfrute de los bienes colectivos que una organización creaba. Este autor definió tres grupos bien identificados: a) grupo privilegiado, b) grupo latente y, c) grupo intermedio, la diferencia radicaba en el disfrute de los bienes colectivos y su costo para la generación de los mismos.

Mientras el grupo privilegiado obtiene mayores ganancias de los bienes colectivos que su contribución individual, los grupos latentes y el intermedio no tienen ese mismo desenvolvimiento para generar bienes colectivos, por lo que su acción colectiva es subóptima. Entonces, Olson ${ }^{41}$ señala que a medida que aumenta el tamaño del grupo, la provisión del bien colectivo se vuelve menos óptima. Solo se puede tener una provisión óptima del bien colectivo si los costos marginales se comparten en exactamente la misma proporción que los beneficios adicionales. En los grupos latentes e intermedios, especialmente en el primero, el costo marginal no se reparte en la misma medida, generando lo que se denomina como free-rider y que no es otra cosa que el actor que no contribuye en la provisión del bien colectivo.

\section{La CAN como grupo sui generis}

Conforme a lo señalado por Casas y Correa en el 2007, la CAN presentó un comportamiento de grupo grande porque los incentivos necesarios para cooperar en búsqueda del interés común no eran posibles en la

39. Olson, La lógica de la acción colectiva: Bienes Públicos y la Teoría de Grupos, 48.

40. Casas y Correa, "QQué pasa con la Comunidad Andina de Naciones-CAN?".

41. Olson, La lógica de la acción colectiva: Bienes Públicos y la Teoría de Grupos. 
medida que el grupo no actuaba como un grupo privilegiado, sino que, por el contrario, había miembros que operaban como free-riders. A pesar del inicio del proceso de reingeniería del 2014, este comportamiento ha continuado a lo largo de los últimos años.

Esta aproximación racional es contrapuesta a la teoría tradicional de la cooperación humana, que presupone que los actores pueden cooperar inclusive en grupos grandes sin tener en consideración sus intereses e incentivos, pero la experiencia empírica demostraría lo contrario, como ha sucedido y sigue sucediendo en la CAN, porque dentro del bloque regional se puede verificar que el costo de generación del bien común no permite que los Estados miembros tengan los incentivos suficientes para avanzar en la profundización del proceso de integración; es decir, sus ganancias no justifican la inversión necesaria (costo marginal). Esto significa que la CAN es un grupo sui generis, pequeño en tamaño pero que opera como un grupo latente según la clasificación de Olson.

El análisis de los problemas de la acción colectiva de la consolidación política en Polonia es un primer ejemplo, en el plano interno, de cómo funciona esta herramienta teórica. La capacidad de los partidos de decidir entre participar políticamente en un contexto de sanción a la fragmentación partidaria o negociar su retiro, estaba constreñida por los incentivos que existían en el marco dado, por lo que la resolución en la acción colectiva era negociar la salida. ${ }^{42}$

Tal y como señalan Casas y Correa lo apreciado en el ejemplo de la participación política en Polonia también se repite en el caso de la CAN, porque la razón primigenia de que el bloque regional se terminará comportando como un grupo latente, se debe a que desde el Acuerdo de Cartagena ha existido un régimen de diferenciación que ha generado una marginalización de los costos que se requieren para conseguir el bien colectivo.

Adicionalmente, en el contexto de los esquemas de integración, esta herramienta teórica se ha usado mucho para analizar la UE y sus procesos decisorios; precisamente, porque esta es una organización de integración que ha pasado de ser de pocos miembros a una que prácticamente reúne

42. Marek Kaminski, "The Collective Action Problems of Political Consolidation: Evidence from Poland", en Collective Choice Essays in Honor of Mancur Olson, ed. Jac C. Heckelman y Denis Coates (Baltimore: Springer, 2003), 76. 
al continente europeo. En el caso de la CAN, la teoría más bien permite entender que su comportamiento genera una acción colectiva subóptima.

La autora Sabine Saurugger ${ }^{43}$ al hacer una revisión de tres distintos documentos de análisis de varios autores (Balne, Chabanet y Wright) concluye que la acción colectiva es una herramienta necesaria para entender procesos de integración que son complejos y, a su vez, limitados, si se mantiene su estudio en la teoría tradicional del regionalismo. Esta misma idea es la que para el caso de la CAN han utilizado Casas y Correa y, en este breve ensayo, corroboramos que sigue siendo vigente luego del inicio de la reingeniería de 2014 .

Conforme lo señalado por Olson, existe mayor efectividad de los grupos relativamente pequeños, privilegiados e intermedios, que se evidencia a partir de la observación y la experiencia, así como de la teoría. ${ }^{44}$ Bajo esta premisa, los participantes de las reuniones que, presumiblemente, tienen interés en llegar a decisiones acertadas, con frecuencia no suelen hacerlo. En el caso de la CAN verificamos que el grupo más bien se comporta como un grupo latente, por ello sus decisiones no son las más óptimas.

La Comunidad Andina es un bloque regional de carácter pequeño conformado por cuatro miembros- lo que le otorga supuestamente ventaja a la hora de tomar decisiones respecto de grupos más amplios. Sin embargo, es preciso considerar que, a pesar de esta ventaja, el bloque no ha avanzado de la forma esperada sobre los temas priorizados en la reingeniería, tal y como se puede verificar en el siguiente cuadro.

\section{Cuadro 1}

Temas priorizados por la Comunidad Andina a partir del proceso de reingeniería

\begin{tabular}{|c|c|}
\hline $\begin{array}{c}\text { Temas prioritarios de la CAN (Decisión Nº } \\
792 \text { del 23 de setiembre de 2013) } \\
\text {-Inicio de Reingeniería de 2014- }\end{array}$ & $\begin{array}{c}\text { Declaración Presidencial de Lima: } 50 \text { Años } \\
\text { de la Comunidad Andina }\end{array}$ \\
\hline & $\begin{array}{l}\text { Avanzar en la construcción de una visión a futuro } \\
\text { de la Comunidad Andina, que priorice el trabajo } \\
\text { orientado a la satisfacción de las necesidades de } \\
\text { sus poblaciones; especialmente, entre otros, en } \\
\text { las siguientes áreas: }\end{array}$ \\
\hline
\end{tabular}

43. Sabine Saurugger, "Review: Collective Action in the European Union: From Interest Group Influence to Participation in Democracy", Comparative Politics 39, n. 4 (2017).

44. Olson, La lógica de la acción colectiva: Bienes Públicos y la Teoría de Grupos, 53. 


\begin{tabular}{|c|c|}
\hline $\begin{array}{l}\text { I) Restructurar el Sistema Andino de Integración } \\
\text { para adecuarlo a las nuevas realidades y retos } \\
\text { del contexto internacional. }\end{array}$ & \\
\hline $\begin{array}{l}\text { 2) Aspectos sociales de la integración: } \\
\text { a) Promover que los ciudadanos puedan viajar, } \\
\text { trabajar, estudiar y residir en cualquier país an- } \\
\text { dino sin discriminación por la nacionalidad, } \\
\text { considerando las particularidades y la seguri- } \\
\text { dad interna de los Países Miembros. } \\
\text { b) Promover la identidad andina }\end{array}$ & $\begin{array}{l}\text { - Programas para la mejora de los servicios públi- } \\
\text { cos de educación y de salud que se brindan a } \\
\text { la población, de acuerdo a lo establecido en el } \\
\text { Acuerdo de Cartagena y la priorización efectua- } \\
\text { da en el proceso de reingeniería. } \\
\text { - Inclusión de las poblaciones vulnerables. } \\
\text { - Promoción de la igualdad de género. } \\
\text { - Participación activa de los pueblos indígenas y } \\
\text { - Librodescendientes en la integración andina. } \\
\text { - Libre movilidad de personas. }\end{array}$ \\
\hline $\begin{array}{l}\text { 3) Profundizar la integración comercial: } \\
\text { a) Internacionalización de las MIPYMES } \\
\text { b) Mecanismos para incrementar el comercio in- } \\
\text { tracomunitario, tales como: } \\
\text { i) Elevación y armonización de estándares de ca- } \\
\text { lidad y reconocimiento mutuo de registros sa- } \\
\text { nitarios. } \\
\text { ii) Facilitación del comercio: aduanas, obstáculos } \\
\text { técnicos al comercio. } \\
\text { c) Exportaciones con valor agregado y encadena- } \\
\text { mientos productivos. } \\
\text { d) Transporte internacional de pasajeros y mer- } \\
\text { cancías. } \\
\text { e) Promoción comercial conjunta. }\end{array}$ & 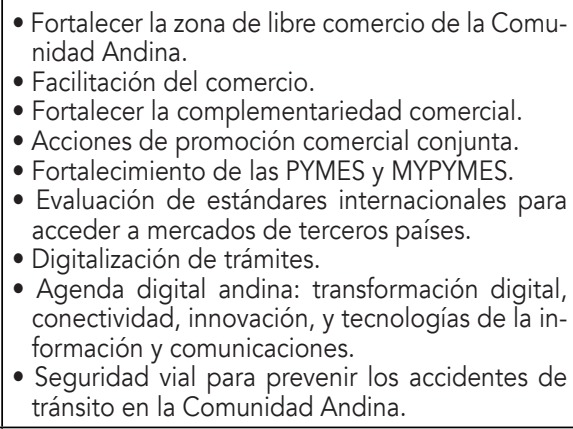 \\
\hline $\begin{array}{l}\text { 4) Interconexión eléctrica: } \\
\text { a) Interconexión intra-regional. } \\
\text { b) Interconexión con otros países de la región. }\end{array}$ & $\begin{array}{l}\text { - Interconexión energética entre países andinos y } \\
\text { otros países de la región. }\end{array}$ \\
\hline $\begin{array}{l}\text { 5) Respecto a una eventual y gradual articulación, } \\
\text { complementación y convergencia de acciones } \\
\text { en el marco de la CAN, MERCOSUR y UNA- } \\
\text { SUR, se recomienda instruir al Secretario Ge- } \\
\text { neral para que: } \\
\text { a) Lleve adelante el mandato de la Declaración } \\
\text { Presidencial de Bogotá de noviembre 2011, } \\
\text { que establece la coordinación de acercamien- } \\
\text { tos con los actores regionales de los procesos } \\
\text { de integración. } \\
\text { b) Presente un informe al CAMRE y a la Comisión } \\
\text { acerca de los elementos comunes, de comple- } \\
\text { mentariedad y diferencias. }\end{array}$ & $\begin{array}{l}\text { Nuestro firme compromiso de fortalecer y dar un } \\
\text { renovado dinamismo al proceso andino de inte- } \\
\text { gración; así como para avanzar hacia un proceso } \\
\text { de diálogo, coordinación y profundización de si- } \\
\text { nergias con los mecanismos regionales y subre- } \\
\text { gionales de integración. Consideramos que, en la } \\
\text { actualidad, la Comunidad Andina debe potenciar } \\
\text { su proyección geográfica hacia otras regiones, y } \\
\text { convertirse en el eje articulador con el resto de } \\
\text { América del Sur. }\end{array}$ \\
\hline
\end{tabular}

Fuente: Decisión Nº 792 del 23 de setiembre de 2013; Declaración Presidencial de Lima: 50 Años de la Comunidad Andina.

Elaboración: autores.

En ese orden de ideas, la CAN, al operar como un grupo latente, no concluye en las decisiones de acción colectiva más óptimas. La gran pregunta radica en qué se debería hacer para modificar esta situación y que el bloque regional pueda ser beneficiario de su reducido tamaño. La respuesta tal vez radica en el segundo presupuesto teórico de la perspectiva de Olson, 
que establece que los grupos más eficientes tienen delimitados sus intereses comunes. La CAN, como se verifica en el cuadro adjunto, ha ido variando sus objetivos, pero una reconfiguración de los mismos a la luz de las necesidades y las contribuciones que los países miembros pueden generar, podría ser la solución para un proceso más eficiente de acción colectiva.

\section{Conclusiones}

El fenómeno de la globalización, tal y como se esbozó en el presente ensayo, puede ser considerado un reto para la teoría tradicional. Tal y como lo señalan Baylis, Smith y Owens, los Estados no están en la capacidad de ser autárquicos y resolver, por sí mismos, las demandas del contexto actual, dada la intensa desterritorialización y la difusión e intensificación de las relaciones sociales a través de las fronteras, lo que hace necesario un esquema distinto para afrontar y resolver esa complejidad existente.

La integración regional, creada bajo el concepto de supranacionalidad, genera una forma de gobernanza en la región para el beneficio de los Estado miembros y, de esa manera, resolver los problemas derivados de la globalización que, de otra forma, no podrían ser resueltos.

La CAN, antes y después del inicio de la reingeniería de 2014, es la respuesta de los países andinos para obtener resultados a los problemas conjuntos; es decir, proveer de bienes colectivos. Es importante subrayar que, a pesar de los importantes logros obtenidos por el grupo, persisten los principales problemas en el bloque que ha presentado a lo largo de su historia.

Tal y como se ha verificado en este ensayo, el comportamiento de la CAN se caracteriza por un accionar como un grupo latente que genera resultados subóptimos y no como un grupo pequeño típico (grupo privilegiado) de la teoría de la acción colectiva. Por lo tanto, se hace necesaria una reflexión profunda sobre el bloque regional a nivel estratégico, particularmente, de sus intereses colectivos y, de esta forma, generar los incentivos necesarios para que la acción colectiva del bloque andino sea más óptima.

El proceso de integración regional podrá considerar pasar por una evolución, más acotada, de la reingeniería, que permita identificar los bienes colectivos que pueden ser suministrados y los costos que los Estados miembros asumirían. La teoría de la acción colectiva puede ser la herramienta conceptual que de luces sobre estos puntos a este proceso. 


\section{Bibliografía}

Barkin, Samuel. International Organization: Theories and Institutions. 2a ed. New York: Pallgrave Mcmillian, 2013.

Baylis, John, Steve Smith y Patricia Owens. The Globalization of World Politics: An Introduction to International Relations. $6^{\text {ta }}$ ed. Oxford: Oxford University Press, 2014.

Casas, Andrés y María Elvira Correa. “QQué pasa con la Comunidad Andina de Naciones-CAN?". Revista Papel Político 12, n. ${ }^{\circ} 2$ (2007): 591-632.

Calduch Rafael. "Las organizaciones internacionales gubernamentales". En Relaciones Internacionales. Madrid, ES: Ediciones Ciencias Sociales, 1991.

Comunidad Andina. Declaración del Consejo Presidencial Andino. 08 de junio de 2020. 〈https://bit.ly/2D5g1Cs〉.

. Declaración del Consejo Consultivo Empresarial Andino. 04 de mayo de 2020. 〈https://bit.ly/34Ba1g3〉.

- Protocolo sobre medidas de prevención sanitaria para las operaciones de Tránsito Aduanero Comunitario entre los Países Miembros de la Comunidad Andina como consecuencia de la pandemia del COVID-19. 02 de mayo de 2020. 〈https://bit. ly/3aZzmBl>.

- Declaración ministerial de Cartagena de Indias con motivo de la celebración de los 50 años de la Integración Andina. 19 de febrero de 2020. 〈https://bit.ly/2YCT$\mathrm{B} 2 \mathrm{Q}$.

Comisión de la Comunidad Andina. Decisión 503 sobre el reconocimiento de documentos nacionales de identificación. 31 de diciembre de 2005.

- Decisión 397 sobre la tarjeta Andina de Migración (TAM). 16 de septiembre de 1996.

. Decisión 536 sobre el marco general para la interconexión subregional de sistemas eléctricos e intercambio intracomunitario de electricidad. 19 de diciembre de 2002. Decisión 757 sobre la vigencia de la Decisión 536. 22 de agosto de 2011.

. Decisión 816 sobre el marco regulatorio para la interconexión subregional de sistemas eléctricos e intercambio intracomunitario de electricidad. 24 de abril de 2011.

. Decisión 672 sobre la modificación de la Decisión 654 sobre el "Marco Regulatorio para la Utilización Comercial del Recurso Órbita Espectro de los Países Miembros". 21 y 28 de septiembre de 2007.

- Decisión 725 sobre la autorización comunitaria para la explotación y comecialización del Recurso Órbita Espectro de los Países Miembros en la posición $67^{\circ}$ Oeste. 10 de diciembre de 2009.

. Decisión 351 el régimen común sobre el derecho de autor y derechos conexos. 17 de diciembre de 1993.

. Decisión 486 sobre el régimen común sobre propiedad industrial. 14 de septiembre de 2000.

. Decisión 345 sobre el régimen común de protección a los derechos de los obtenedores de variedades vegetales. 21 de octubre de 1993. 
. Decisión 391 sobre el régimen común sobre acceso a los recursos genéticos. 02 de julio de 1996.

- Decisión 291 sobre el régimen común de tratamiento a los capitales extranjeros y sobre marcas, patentes, licencias y regalías. 21 de marzo de 1991.

- Decisión 632 sobre la aclaración del segundo párrafo del artículo 266 de la Decisión 486 de 2000. 06 de abril de 2006.

. Decisión 689 sobre la adecuación de determinados artículos de la decisión 486. 13 de agosto de 2008.

- Decisión 366 sobre la modificación de la disposición transitoria tercera de la Decisión 345. 26 de noviembre de 1994.

- Decisión 423 sobre la modificación de la octava disposición transitoria de la decisión 391.11 de noviembre de 1997.

. Decisión 448 sobre la modificación de la octava disposición transitoria de la decisión 391. 11 de diciembre de 1998.

- Decisión 376 sobre la aplicación de las Tablas Aduaneras del Sistema Andino de Franjas de Precios. 18 de abril de 1995.

. Decisión 419 sobre la modificación de la Decisión 376. 30 de julio de 1997.

. Decisión 515 sobre el sistema andino de sanidad agropecuaria. 08 de marzo de 2002.

- Decisión 516 sobre la armonización de legislaciones en materia de productos cosméticos. 08 de marzo de 2002.

- Decisión 706 sobre la armonización de legislaciones en materia de productos de higiene doméstica y productos absorbentes de higiene personal. 9 y 10 de diciembre de 2008.

Cram, Laura. "The EU institutions and Collective Action: Constructing a European Interest". En Collective Action in the European Union: Interests and the new politics of associability, editado por Justin Greenwood y Mark Aspinwall, 63-80. New York, EE.UU: Routledge Editores, 1998.

Held, David, Anthony McGrew, David Goldblatt,y Jonathan Perraton,. Global Transformations: Politics, Economics and Culture. Stanford: Stanford University Press, 1999.

Kaminski, Marek. "The Collective Action Problems of Political Consolidation: Evidence from Poland". En Collective Choice Essays in Honor of Mancur Olson, editado por Jac C. Heckelman y Denis Coates, 71-89. Baltimore: Springer, 2003.

Olson, Mancur. La lógica de la acción colectiva: Bienes Públicos y la Teoría de Grupos. México: Limusa Noriega Editores, 1992.

Nicoli, Franceso. "Neofunctionalism revisited: Integration Theory and varieties of outcomes in the Eurocrisis". Journal of European Integration 41, (2019). doi: 10.1080/07036337.2019.1670658.

Rosenau, James. Along the Domestic-Foreign Frontier: Exploring Governance in a Turbulent World. Cambridge. Cambridge University Press, 1997. 
Saurugger, Sabine. "Review: Collective Action in the European Union: From Interest Group Influence to Participation in Democracy". Comparative Politics 39, n. ${ }^{\circ}$ 4 (2017).

Scheuerman, William. "Globalization". The Stanford Encyclopedia of Philosophy winter 2018 Edition, (2018). ‘https://stanford.io/2G50zr6».

CAN. "CAN cifras 2019". Comunidad Andina de Naciones, Perú. 2019. «https://bit. ly/2EAlyBJ

Tussie, Diana. "El coronavirus va a profundizar la resistencia a la globalización". La Nación. 05 de abril de 2020. 〈https://bit.ly/2G51BDu〉.

Weiss, Thomas. Global Governance: Why? What? Whither? New York: Polity, 2013. 